\title{
Wohnungsfrage(n) - Multiple Perspektiven auf ein zentrales Themenfeld sozialwissenschaftlicher Forschung konkretisiert am Beispiel Kurzzeitvermietung
}

*christian.smigiel@sbg.ac.at, Fachbereich Geographie und Geologie, Universität Salzburg

eingereicht am: 18.04.2021, akzeptiert am: 21.06.2021

Die Wohnungsfrage wird als eine der zentralen sozialen Fragen des 21. Jahrhunderts bezeichnet. Sowohl in der Öffentlichkeit als auch in verschiedenen Sozialwissenschaften wird sie intensiv diskutiert und erforscht. Vor diesem Hintergrund liefert der vorliegende Beitrag einen kritisch-konzeptionellen Überblick, ordnet die thematische Vielfalt der Wohnungsfrage und konkretisiert die Multidimensionalität anhand aktueller Forschungserkenntnisse zu Kurzzeitvermietungen (Airbnb und Co.).

Keywords: Wohnen, sozialräumliche Ungleichheit, kritische Stadtforschung, Kurzzeitvermietung

\section{Housing question(s) revisited - multiple perspectives on a core issue of social sciences. The case of short-term rentals}

The housing question is one of the most urgent social issues of the 21 st century. Therefore, it is a widely discussed topic in public debates as well as many social sciences. This paper provides a critical overview how to conceptualize the housing question. Furthermore, it discusses multiple dimensions of housing and uses the example of short-term rentals (Airbnb anc Co.) in order to demonstrate its overall impact on urban fabric and everyday life.

Keywords: housing, socio-spatial inequalities, critical urban studies, short-term rentals

\section{$1 \quad$ Einleitung}

„Wohnst Du noch oder lebst Du schon?" So lautete Anfang der 2000er-Jahre einer der bekanntesten Werbesprüche eines schwedischen Einrichtungshauses. Gegenwärtig bedarf es keiner Marketingaktivitäten, um die Bedeutung des Wohnens in den Mittelpunkt des öffentlichen Interesses zu rücken. Steigende Wohnpreise, Wohnraumknappheit, Verdrängung von Bewohner*innen oder Leerstand sind Schlagworte, die die Wohnungsfrage $(n)^{1}$ in vielen urbanen und ruralen Kontexten bereits seit längerer Zeit prägen. Darüber hinaus verschärft die gegenwärtige Corona-Pandemie vielerorts die Wohnungskrise. ${ }^{2}$ All diese Facetten

\footnotetext{
Eine umfangreiche Erörterung der Vielschichtigkeit und Wechselwirkung von Wohnungsfragen findet sich u.a. bei Fezer et. al 2017 und Schönig \& Vollmer 2020. Im Folgenden werde ich aus stilistischen Gründen „nur“ von der Wohnungsfrage sprechen. Der Plural sei hier aber stets mitgedacht.

2 Vor diesem Hintergrund erklärt sich auch der vielstimmige öf-
}

unterstreichen die Spannbreite dieses Megathemas. Zugleich wird deutlich, wie vielfältig, ja in gewisser Weise auch unübersichtlich und widersprüchlich der Themenbereich „Wohnen“ ist.

Im folgenden Beitrag geht es darum, das komplexe und interdisziplinäre Forschungsfeld Wohnen, welches von unterschiedlichen sozialwissenschaftlichen Disziplinen (Ethnographie, Geographie, Ökonomie, Planungswissenschaften, Politikwissenschaft, Soziologie etc.) untersucht wird, zu skizzieren und greifbar zu machen. Nach einer kurzen Erörterung der gesellschaftlichen Bedeutung und gleichzeitigen Konflikthaftigkeit des Wohnens in Kapitel 2, wird die ewig junge Wohnungsfrage als heuristisches Werkzeug

fentliche Diskurs um das Wohnen, der (auch international) von Debatten um mehr (sozialen) Wohnbau in politischen Auseinandersetzungen, Ausstellungen, Dokumentarfilmen über Diskussionen um stärkere Kontrollen und Beschränkungen von Mietpreisen bis hin zu neuen Mieter*innenbewegungen reicht (Fezer et al. 2017; Rink \& Vollmer 2019; Holm 2020). 
verwendet, um zentrale thematische Dimensionen des Wohnens in Kapitel 3 herauszuarbeiten, die ein tieferes Verständnis des Wohnens ermöglichen. Anschließend werden die genannten Dimensionen der Wohnungsfrage am Beispiel Kurzzeitvermietungen (Airbnb und Co.) konkretisiert. Das Ziel ist es, einen strukturierten Überblick über einen zentralen Forschungsbereich sozialwissenschaftlicher Forschung zu geben, der sowohl einen konzeptionellen Rahmen anbietet als auch einen empirischen Einblick gewährt. Neben diesem fachwissenschaftlichen Ein- und Überblick ist die Wohnungsfrage auch in bildungspolitischer Hinsicht von Bedeutung und dürfte als Querschnittsthema nicht zuletzt für den GW-Unterricht in Zukunft an Relevanz gewinnen.

\section{Gesellschaftliche Relevanz und Kon- flikthaftigkeit}

Leistbares, sicheres und angenehmes Wohnen ist ein zentrales menschliches Grundbedürfnis. Diese Erkenntnis ist Bestandteil der allgemeinen UN-Menschenrechtscharta, der europäischen Sozialcharta und vieler nationaler Verfassungen (Hubeli 2020: 72). Allerdings ist dies (fast immer) nur ein abstrakter politischer Anspruch und kein individuell einklagbares Grundrecht. ${ }^{3}$ Dieser Widerspruch begegnet uns überall im Alltag, wenn wir bspw. eine Wohnung suchen. Gesellschaftliche Relevanz und Konflikthaftigkeit des Wohnens zeigen sich auch im Zusammenspiel mit anderen Daseinsgrundfunktionen wie Arbeit, Bildung, Gesundheit oder Mobilität. Wo wir wohnen, bestimmt unser Mobilitätsverhalten. Der Wohnort entscheidet darüber, wie viel Zeit wir für unsere Arbeits-, Ausbildungs- und Freizeitwege benötigen. Diese vielfältigen Mobilitätsformen haben wiederum großen Einfluss auf ökologische Aspekte wie Luftverschmutzung, Wasserqualität, Lärm etc. und sind somit auch in klimapolitischer Hinsicht von Bedeutung.

Dass der Wohnort auch für unsere Gesundheit entscheidend ist, haben bereits frühe sozialwissenschaftliche Klassiker eindrucksvoll aufgezeigt, in dem sie auf die katastrophalen hygienischen Wohnbedingungen und deren strukturelle Ursachen zu Beginn des Industriekapitalismus hingewiesen haben (Engels 1970 [1845]). Man muss aber nicht 180 Jahre zurückgehen, um die strukturellen Zusammenhänge von Wohnungsfrage und Gesundheit herauszuarbeiten. Aktuelle Forschungen, die sich mit der gegenwärtigen

\footnotetext{
3 Ein „Recht auf Wohnen“ findet sich in der österreichischen Verfassung oder dem deutschen Grundgesetz als Staatszielbestimmung. Ein subjektives „Recht auf Wohnen“ ist damit nicht verbunden. (Deutscher Bundestag 2019: 5).
}

COVID-19 Pandemie beschäftigen, veranschaulichen, dass Infektionsgefahr, schwerwiegende Krankheitsverläufe oder soziale Benachteiligungen ganz wesentlich mit dem Wohnort zusammenhängen können (Plümper \& Neumayer 2020).

Der Wohnort hat zudem maßgebliche Auswirkungen auf den Bildungsweg und auch auf den Bildungserfolg von Kindern. Dies beginnt bereits mit der Wahl der "passenden“ Volksschule/ Grundschule, die möglichst in einem Einzugsgebiet mit gehobenem sozioökonomischen Status liegen soll. Ein Indiz dieser Entwicklung ist die Zunahme schulischer Segregation bzw. homogener Schulwelten (Parade \& Heinzel 2020: 196). Dieser Trend führt zwar nicht zwangsläufig zu homogenen Wohnquartieren, bzw. residentielle Segregation ${ }^{4}$ führt nicht automatisch zu schulischer Segregation, aber aktuelle Langzeitstudien aus 74 deutschen Städten belegen, dass gerade die soziale Segregation bei Kindern und vor allem die soziale Segregation von armen Haushalten mit Kindern stark zugenommen hat (Helbig \& Jähnen 2018: 45).

Dies wirft wiederum Ungleichheits- bzw. Gerechtigkeitsfragen auf, die im Zusammenhang mit der Wohnungsfrage in jüngster Zeit auch verstärkt geäußert werden. Prominent werden diese politischen Forderungen in Berlin diskutiert, wo sich auch aufgrund der exorbitant gestiegenen Mietpreise und zunehmender Wohnungsnot ein breites gesellschaftliches Bündnis zusammengefunden hat (Holm 2020). Neue politische Mieter*innenbewegungen finden sich aber nicht nur in der deutschen Hauptstadt, sondern auch in anderen Städten, die mit ähnlichen Problemlagen zu kämpfen haben. Gefordert werden vielerorts konkret-fundamentale Veränderungen kapitalistischer Wohnraumproduktion, die auf neue Wohnformen, neue Verteilungsmodelle und neue Wohnungsmarktstrukturen abzielen und somit die Multidimensionalität der Wohnungsfrage verdeutlichen (Rink \& Vollmer 2019: 340).

\section{Dimensionen der Wohnungsfrage}

Das vorangegangene Kapitel hat neben der gesellschaftlichen Relevanz, Widersprüchlichkeit und Konflikthaftigkeit auch die Komplexität der Wohnungsfrage deutlich gemacht. Vor diesem Hintergrund soll im Folgenden eine konzeptionelle Einordung der

\footnotetext{
Residentielle Segregation beschreibt die unfreiwillige Trennung von sozialen Gruppen oder Individuen basierend auf dem Wohnstandort, die das Ergebnis von strukturellen Ungleichheiten auf dem Wohnungsmarkt, Einkommensungleichheiten, oder politischer Steuerung ist bzw. aus der lebensstilorientierten Wahl des Wohnstandortes resultiert (Bürkner 2018).
} 
Wohnungsfrage vorgenommen werden. Angesichts der Komplexität der Wohnungsfrage ist es nicht der Anspruch einen allumfassenden Überblick bezüglich der Multidimensionalität der Wohnungsfrage zu geben. ${ }^{5}$ Vielmehr beschränke ich mich im folgenden $\mathrm{Ka}-$ pitel auf vier Dimensionen, die jedoch anschlussfähig an breitere Debatten in der Wohnungsforschung sind (Schönig \& Vollmer 2020: 11 ff.). Die vier vorgestellten Dimensionen ermöglichen eine integrative Betrachtung unterschiedlicher Thematiken, die mit der Wohnungsfrage in unterschiedlichen räumlichen und zeitlichen Kontexten verbunden sind. Gleichzeitig sind die politökonomische, die rechtlich-planerische, die geographische und die ökologische Dimension untereinander verwoben; sie bedingen einander. Das heißt, die vier skizzierten Dimensionen können als ein Kontinuum verstanden werden, in dem sich die Komplexität der Wohnungsfrage spiegelt. Ein weiteres Anliegen des Kapitels ist es, eine Grundlage zu schaffen, von der aus konkrete Macht- und Kräfteverhältnisse der Wohnungsfrage erörtert werden (siehe Kapitel 4). In diesem Sinne helfen uns die vier diskutierten Dimensionen zu verstehen, wie Wohnen produziert, organisiert und gelebt wird.

\subsection{Politökonomische Dimension}

$\mathrm{Zu}$ Beginn vieler Erörterungen der Wohnungsfrage steht die Feststellung, dass "Wohnen“ in unserer gegenwärtigen gesellschaftlichen Verfasstheit einem grundsätzlichen Widerspruch unterliegt. Worin liegt der Widerspruch begründet? Einerseits ist „Wohnen" ein Gut, dass in Kapitalkreisläufe eingebettet ist und von Kapitalinteressen bestimmt wird. Wohnraum wird als Geldanlage von Privatpersonen gebaut, ge- und verkauft oder genutzt. Gleiches gilt auch für institutionelle Anleger*innen (z. B. Kreditinstitute, Pensionskassen, Versicherungen, Fonds), die Kapital in Immobilien anlegen, mit Wohnraum spekulieren, diesen als Sicherheit verwenden bzw. damit handeln. Wohnraum hat somit einen Tauschwert, der zudem nicht mehr nur lokal gehandelt wird. ${ }^{6}$ Andererseits ist Wohnen ein Grundbedürfnis, das gebraucht und genutzt wird. Wohnen hat einen lokalen Gebrauchswert, der nicht substituierbar ist. Dieser Doppelcharakter der Ware Wohnen ist nicht nur durch Interessensge-

\footnotetext{
Eine ausführliche und lesenswerte Erörterung verschiedener Dimensionen der Wohnungsfrage findet sich in einem aktuellen Sammelband, der Texte zur Wohnungsfrage vereint (Schönig \& Vollmer 2020). 6 Spätestens seit der Wirtschafts- und Finanzkrise von 2008 ist der globale Charakter der Ware Wohnen auch einer breiten Öffentlichkeit ein Begriff. Die damaligen Verwerfungen auf den internationalen Finanzmärkten wurden nicht zuletzt durch den weltweiten Handel und Verkauf von Hypothekenkrediten ausgelöst (Padberg \& Schraven 2015).
}

gensätze geprägt (Privatanleger*innen bzw. institutionelle Anleger*innen streben grundsätzlich höhere Wohnpreise an; die Nutzer*innenseite präferiert stabile oder sinkende Preise für Miete oder Haus), sondern wird von unterschiedlichen Machtpositionen geführt (Holm 2011: 16).

In diesem Grundkonflikt kommt der öffentlichen Hand eine entscheidende Rolle zu. Einerseits versuchen politische Institutionen in Wohlfahrtsstaaten schon seit vielen Jahrzehnten diesen Grundkonflikt zumindest abzuschwächen, indem sie z. B. Mieter*innen gesetzlich stärken, Wohnraum für einkommensschwache Haushalte bereitstellen, steuerlich subventionieren oder Bauland für geförderten Wohnbau reservieren (Chorherr 2019: 12). Andererseits sind die meisten Strategien und Lösungen auch in Österreich und Deutschland entweder räumlich, zeitlich oder inhaltlich befristet (Schönig \& Vollmer 2020: 9). Öffentlicher Wohnbau bzw. soziale Wohnraumversorgung $^{7}$ ist hierfür ein aufschlussreiches Beispiel. Zwar zählt er zum Erbe sozialstaatlicher Errungenschaften des 20. Jahrhunderts, gleichzeitig gibt es regional sehr große Unterschiede, was Anzahl und Ausgestaltung öffentlicher Wohnraumversorgung anbetrifft. ${ }^{8} \mathrm{Zu}-$ dem ist vor allem der sogenannte soziale Wohnbau in den letzten Jahrzehnten stark rückläufig und darüber hinaus per Definition (bspw. in Deutschland) mit einem Ablaufdatum versehen, d.h. er ist zeitlich befristet und verliert mit Fristende seine gedeckelten Mietpreise, was steigende Mieten und Verdrängungsprozesse nach sich zieht (Kockelkorn 2017: 110). Die Befristung öffentlich geförderten Wohnbaus zeigt sich auch in anderer Hinsicht. Geförderter Wohnbau wird in Österreich und Deutschland auch von privaten, gewinnorientierten Wohnbaufirmen betrieben, mit der Perspektive diesen nach zeitlichem Ablauf der Befristungen zu verkaufen oder auf dem privaten Wohnungsmarkt anbieten zu können (Van Hametner et al. 2019: 239).

\footnotetext{
Unter öffentlichem Wohnbau verstehe ich die Gesamtheit des Wohnraums, der von der öffentlichen Hand geschaffen wird. Wichtige Akteure sind u.a. gemeinnützige Bauvereinigungen, Genossenschaften oder kommunale Wohnungsunternehmen. Darüber hinaus gibt es auch (neue) private genossenschaftliche Organisationen (bspw. Mietshäusersyndikat), die Wohnraum gezielt abseits des nicht regulierten, freien Wohnungsmarkts errichten und dauerhaft anbieten, um es den Zwängen des kommerziellen Wohnungsmarkts zu entziehen.

8 Mit Blick auf Österreich lassen sich die regionalen Unterschiede am „roten Wien“ mit seiner langen Tradition des öffentlichen Wohnbaus und der sozialen Wohnraumversorgung und Städten wie Salzburg mit seiner dominanten Privateigentümer*innenstruktur und der marginalen Rolle öffentlicher Wohnraumversorgung exemplarisch ablesen. Gleichwohl muss angemerkt werden, dass sich auch die Stadt Wien aus dem Neubau von Gemeindewohnungen für einige Jahrzehnte zurückgezogen hatte (Zeller et al. 2018).
} 
Dieser Exkurs zum öffentlichen Wohnbau deutet einen grundsätzlichen Aspekt an: Die öffentliche Hand folgt den ökonomischen und rechtlichen Logiken des (Wohnungs)Marktes. Dies sieht man bspw. daran, dass gemeinnützige Bauvereinigungen oder Kommunen „marktübliche“ Grundstückspreise bezahlen (müssen), wenn sie Bauland erwerben, wodurch auch die öffentliche Hand mit an der Preisschraube dreht. Die Funktionalität des Wohnungsmarkts wird hingegen nicht hinterfragt, obgleich der (Wohnungs)Markt für hohe Wohnpreise und andere Verwerfungen mit verantwortlich ist, da nur die Verknappung einer Ware auf einem Markt zu hohen Gewinnen führt (Fezer et al. 2017: 13). Gleiches gilt in besonderem Maße für das knappe Gut Boden, dessen Preise in vielen europäischen und nordamerikanischen Metropolen in den letzten Jahrzehnten um das Hundertfache gestiegen sind, während Löhne und Inflation wesentlich geringere Steigerungsraten aufweisen (Hubeli 2020: 46). Die Gewinne aus diesen Wertsteigerungen des Bodens, die sich bspw. aufgrund steigender Nachfrage, Verknappung oder Umwidmungen ${ }^{9}$ von Flächen ergeben können, sind private Gewinne, die lediglich den Bodeneigentümer*innen zu Gute kommen. Abgaben auf diese Gewinne gibt es bspw. in Österreich kaum (Mayer 2020: 81). Vielmehr wird Bodeneigentum steuerlich begünstigt behandelt und darüber hinaus erfolgt oftmals eine öffentlich geförderte Wertsteigerung, in dem die öffentliche Hand Infrastruktur bereitstellt, wodurch der Wert des Grundstücks maßgeblich steigt.

Eine Verschärfung der Wohnungsfrage lässt sich vor allem seit Anfang der 2000er-Jahre beobachten. Miet-, Haus- und Bodenpreise steigen auch in österreichischen und deutschen Städten teilweise exorbitant (Amann \& Lugner 2016: 46; Egner \& Rink 2020: 310). ${ }^{10}$ Der Prozess der Finanzialisierung ist hierfür ein wesentliches Kriterium. Finanzialisierung im Bereich Wohnen beschreibt eine Entwicklung, wodurch Wohnen als Finanzgut zunehmende Bedeutung gewinnt (Aalbers 2019). Wohnen, sowie Grund und Boden wurden zu Finanzinstrumenten, die (als Eigentumstitel) gehandelt und veräußert und so zu einer profitablen Geldanlage gemacht werden. Neue Marktakteure (diverse Immobilienfonds), politischrechtliche Entscheidungen und neue Praktiken haben seit Anfang der 2000er-Jahre zu dieser Entwicklung beigetragen. Die Spannbreite der Akteure reicht von

\footnotetext{
9 Allein die Umwidmung von Grünland in Bauland führt zu einer exorbitanten Erhöhung des Bodenpreises.

10 In Österreich stiegen die Bodenpreise zwischen 2015 und 2018 um 76\%, während die Nettojahreseinkommen im gleichen Zeitraum sich um lediglich 9\% erhöhten (Mayer \& Ritter 2020: 16). Hierbei handelt sich wohlgemerkt nur um Durchschnittswerte.
}

transnationalen Immobilienkonzernen und Immobilienfonds bis hin zu privaten Kleinanleger*innen, die eine Vorsorgewohnung kaufen (Aigner 2020). Vor allem bei Ersteren liegt das Hauptaugenmerk auf der Wertsteigerung und nicht auf der Vermietungspraxis (Hubeli 2020). Eingebettet sind diese Praktiken in besagten Prozess der Finanzialisierung, der durch eine Liberalisierung und Deregulierung der Finanzmärkte in den 1990er-Jahren angestoßen wurde, wodurch zum einen neue Marktakteure erst entstehen konnten und zum anderen Immobilien erst zu einem attraktiven, weil beweglichen Kapitalanlagefeld gemacht wurden (Heeg 2013).

Ein weiterer Baustein, der zu Preisanstiegen und der Verschärfung der Wohnungsfrage beigetragen hat, ist die Privatisierung öffentlicher Wohnungsbestände in vielen europäischen Stadtregionen (Elsinga et al. 2014: 410). Anfang der 2000er-Jahre machten umfangreiche Veräußerungen von öffentlichen Wohnungsbeständen auch in Österreich und Deutschland Schlagzeilen (Claßen \& Zander 2010; Streimelweger 2010). Zahlreiche Kommunen (bspw. Berlin und Dresden), öffentliche Institutionen (ÖBB und DB), aber auch private Konzerne wie Thyssen Krupp und E.ON verkauften in den 2000er-Jahren mehrere zehntausende Wohneinheiten vor allem an private Immobilienkonzerne und Immobilienfonds oder privatisierten öffentliche Wohnbauträger, wodurch die öffentliche Hand zwar kurzfristig Einnahmen erhielt, mittel- und langfristig aber Gestaltungsspielraum abgab, die den späteren Anstieg der Wohnpreise mit initiiert hat (Claßen \& Zander 2010: 381; Kockelkorn 2017: 112). ${ }^{11}$ Mittlerweile hat man von dieser Praxis zwar Abstand genommen, gleichwohl gibt es andere Formen der Kommodifizierung des Wohnens, die deutlich machen, dass sich die Einbindung privatwirtschaftlicher Akteure in der Wohnungsfrage vertieft hat (siehe Kapitel 4 am Beispiel Kurzzeitvermietungen).

\section{Löst Wohneigentum für alle die Wohnungsfrage?}

Ein wichtiger Aspekt der Wohnungsfrage, der in keiner wohnungspolitischen Auseinandersetzung fehlt, betrifft die Kontroverse um Miete vs. Eigentum. Wie unterschiedlich diese Frage beantwortet wird, zeigt bereits ein Blick auf die Eigentumsverhältnisse in Europa. Während im südlichen und östlichen Europa die Wohneigentumsquoten aus unterschiedlichen Gründen bei $80-90 \%$ liegen, betragen sie in

\footnotetext{
1 Hinzukommen Privatisierungen öffentlicher Liegenschaften, die indirekt mit der Wohnungsfrage zusammenhängen und den Gestaltungsspielraum der öffentlichen Hand massiv einschränkt haben (Silomon-Pflug 2018).
} 
Schweden oder Dänemark, aber auch in Österreich und Deutschland nur 40-50\%. Wenngleich die nationalen Durchschnittswerte nur einen Teil der lokalen Wirklichkeiten abbilden, dominiert in den letztgenannten Ländern die Miete. Dies hat wiederum Einfluss auf die Leistbarkeit oder den Zugang zum Wohnungsmarkt und sorgt somit für unterschiedliche soziale Mobilität (Scanlon et al. 2014: 7 ff.). Gleichzeitig bedeutet Eigentum langfristige Kapitalbindung und vor allem bei kapitalschwachen Haushalten auch Verschuldung, wie die Subprime Crisis von 2008 (siehe Fußnote 6) oder Margaret Thatchers „Right to buy"-Politik der 1980er-Jahre in Großbritannien gezeigt haben (Whitehead 2014). Es ist somit mehr als fraglich, ob Wohneigentum für breite soziale Schichten eine adäquate Lösung in ökonomischer und sozialer Hinsicht darstellt oder ob Wohneigentum nicht vielmehr zur Vertiefung sozialräumlicher Disparitäten beiträgt.

\subsection{Rechtlich-Planerische Dimension}

Eine auch in der Wohnungsforschung wenig beachtete Dimension betrifft die Rolle des Rechts als politisch-räumliches Instrument sowie die Funktion des Rechts als maßgeblicher Struktur- und Machtfaktor in der Wohnungsfrage. Was ist damit gemeint? Die Juristin Katharina Pistor geht in ihrem Buch „Code des Kapitals" auf die lange Tradition des Rechts und spezieller Rechtsordnungen ein, die Güter wie Wohnraum, Grund und Boden erst handelbar machen, indem sie sie rechtlich kodieren. Sie nennt in diesem Zusammenhang u. a. das Eigentumsrecht, aber auch das Vertrags- und das Kreditsicherungsrecht (Pistor 2020: 17). Mittels dieser Rechtsordnungen werden bspw. Eigentümer*innen von Grund und Boden gegenüber anderen Ansprüchen quasi dauerhaft geschützt, ihre Anliegen werden als prioritär eingestuft und sie haben die Möglichkeit ihr Eigentum notfalls in Geld einzutauschen. Durch vielfältige Codierungspraktiken werden Vermögenswerte geschaffen, geschützt und können somit ungestört vermehrt werden. Ein wichtiger Aspekt gerade im Kontext der Wohnungsfrage ist die Mobilität bzw. Universalität von Rechtsordnungen, die auch das weltweite Investieren in Wohnraum und die angesprochene Finanzialisierung des Wohnens ermöglichen. Konkret können sich global agierende Immobilienunternehmen ihren Unternehmensstandort dahingehend aussuchen, wo die für sie passenden Rechtsordnungen vorliegen, wodurch steuerliche Abgaben und Gewinne nicht in dem Land versteuert werden, wo das Immobiliengeschäft getätigt wird (Smigiel 2016).

Gesetze und rechtliche Normen haben zudem eine weitere Eigenschaft. Sie „neutralisieren“ den politi- schen Charakter von Regierungshandeln, der sich folglich hinter scheinbar selbstverständlichen Rechtsnormen verbirgt (Moore \& Schindler 2017: 66). Gleichzeitig sorgen sie auch in zeitlicher Hinsicht für eine Verstetigung politischer Weichenstellungen, in dem sie politische Richtungsentscheidungen institutionell verankern. Zwei Beispiele sollen das rechtlich-politische Zusammenspiel im Kontext der Wohnungsfrage verdeutlichen. Sie unterstreichen zudem die Langzeitwirkung dieses regulierenden Nexus. 1990 wurde in der Bundesrepublik Deutschland mit dem Steuerreformgesetz die Wohnungsgemeinnützigkeit aufgehoben, wodurch mehrere Millionen Wohnungen aus der Mietpreisbindung herausfielen und 1200 Genossenschaften ihre steuerlichen Vorteile gegenüber privaten Immobilienunternehmen verloren (Kockelmann 2017: 115). Dem war eine langjährige Diskreditierung der Wohngemeinnützigkeit seit den 1970er-Jahren vorausgegangen, mit der der gesamte öffentliche Wohnungsbau sukzessive eine neue Bedeutung zugewiesen bekam. Entgegen der vorherigen Ausrichtung war sozialer Wohnungsbau nun kein gesamtgesellschaftliches Anliegen, sondern wurde zu einem expliziten Instrument der Armutsbekämpfung deklariert (Kockelmann 2017: 118). Das zweite Beispiel betrifft die steuerlich subventionierte Eigentumsbildung, die nicht nur in der BRD die Wohnungspolitik prägt. Neben rechtlicher Priorisierung des Eigentums gibt es auch steuerliche Sonderabschreibungen, die bspw. im Zuge der deutschen Einheit im Fördergebietsgesetz festgelegt wurden. Hiernach konnten sich Kapitalanleger*innen bei Immobilieneigentumserwerb in ostdeutschen Bundesländern 50\% der getätigten Kosten rückerstatten lassen (Aring \& Herfert 2001: 46). Dies führte einerseits zu einem Bauboom im Umland ostdeutscher Städte (Suburbanisierung) und zu einem massiven Aufkauf von Immobilien (inklusive Renovierung) in ostdeutschen Innenstädten vor allem durch westdeutsche Kapitalanleger*innen. ${ }^{12}$ In Österreich hat es auf der Bundesebene keine derart marktkonformen gesetzlichen Maßnahmen gegeben. Gleichwohl hat auch hier eine schleichende Neoliberalisierung der Wohnungsfrage Einzug gehalten, die auch rechtlich verankert wurde. Dies lässt sich an den Veränderungen im Mietrechtsgesetz in den 1990er-Jahren, der Kürzung der rechtlich festgelegten Wohnbauförderung und dem Wandel von Objekt- zu Subjektförderung ${ }^{13}$ beobachten (Zeller et al. 2018).

\footnotetext{
12 Kapitalbildung war Einwohner*innen der ehemaligen DDR von 1949-1989 kaum möglich gewesen.

13 Unter Objektförderung lassen sich alle direkten Förderungen für den Bau bzw. Instandhaltung von Wohnraum zusammenfassen. Subjektförderung ist eine Förderform, die vor allem als Geldoder Kreditleistung unterschiedlichen Haushalten zukommt und somit indirekt den Wohnbau beeinflusst. Sowohl in Österreich als
} 
Als Fazit dieser Ausführungen kann festgehalten werden, dass eine generelle Reskalierung nach unten bzw. eine Regionalisierung der Wohnungsfrage stattgefunden hat (im österreichischen Kontext hat die Bundesebene Kompetenzen an die Bundesländer übertragen) (Schönig \& Vollmer 2020: 13). In Deutschland wiederum wurden zahlreiche öffentliche Wohnraumbestände nicht nur an private Immobilienakteure veräußert, sondern auch in kommunale Tochterunternehmen ausgegliedert. Dies hat zu einer Fragmentierung der Wohnungsfrage beigetragen, die nun weniger gesamtstaatlich, sondern vielmehr lokal gemanagt wird.

Auf regional-lokaler Ebene kommt zudem der Stadt- und Regionalplanung eine wichtige Rolle in der Wohnungsfrage zu. Obwohl Stadt- und Regionalplanung eher strategisch ausgerichtet ist, mittel- bis langfristige regionale Entwicklungskonzepte ausarbeitet, Flächenwidmungs- oder Bebauungspläne entwirft sowie Umweltprüfungen durchführt und somit eher langfristige Weichenstellungen vornimmt, gibt es aktuelle Entwicklungen in der Wohnungsfrage, die auch kurzfristige Lösungen möglich machen (siehe Kapitel 4.3). Insbesondere Zweit- und Ferienwohngebiete sind zu einem Streitpunkt in vielen österreichischen Städten und Gemeinden geworden, was zumindest teilweise relativ kurzfristige Anpassungen der Raumordnungen nach sich zieht. Die Schärfe und Entwicklung der jeweiligen Planungsinstrumente oder rechtlichen Verordnungen mit denen diese und andere raumrelevante Sachverhalte angegangen werden, hängt stark von der politischen und öffentlichen Meinungsbildung ab. Diese Meinungsbildung findet nicht nur in den betroffenen Gemeinden oder Kommunen statt. Vielmehr ist dies ein multiskalarer Prozess, in dem unterschiedliche Interessen aufeinandertreffen (siehe Kapitel 4).

\subsection{Geographische Dimension}

Die Wohnungsfrage hat seit jeher auch eine geographische Dimension. Soll heißen, die Wohnungsfrage äußert sich je nach räumlichem Kontext unterschiedlich. Gleichzeitig wird die Wohnungsfrage nicht nur durch die spezifischen Pfadabhängigkeiten einer bestimmten Stadt, Region oder eines Nationalstaats geprägt, sondern Wohnen ist selbst wesentlicher Faktor, der diese bestimmten räumlichen Wirklichkeiten (mit)produziert (Fezer et al. 2017: 14). Dieses wird im Folgenden anhand paradigmatischer Entwicklungen und Prozesse (Suburbia, Großwohnsiedlungen, Gentrifizierung, Segregation) skizziert.

auch in Deutschland ist seit mehreren Jahrzehnten zu beobachten, dass die direkten Maßnahmen im Wohnbau seitens der öffentlichen Hand zurückgehen, während die Unterstützungsleistungen (Wohnbeihilfe, Wohngeld) zunehmen.
Die unterschiedlichen räumlichen Ausprägungen der Wohnungsfrage stehen seit langem im Mittelpunkt der (sozial)wissenschaftlichen (Stadt) Forschung. Bereits im 19. Jahrhundert wird die Wohnungsfrage überwiegend an Städten diskutiert. So erörtert Friedrich Engels in seinen bahnbrechenden Studien strukturelle Ursachen und die Lage der englischen Arbeiter*innenklasse in London, Manchester, Edinburgh, Glasgow und anderen Städten des industriekapitalistischen Großbritannien und zeigt somit die Zusammenhänge zwischen Wohnungsmisere, Industrialisierung und Urbanisierung (Engels 1845, 1872). Aber auch die frühen sozialräumlichen Kartierungen von Industriellen wie Charles Booth erörtern die Wohnsituation in Städten und liefern erste Erkenntnisse zu sozialer Segregation. Diese Liste ließe sich weiter fortsetzen. Es zeigt sich jeweils, dass mit dem Fokus auf Städte nicht nur wichtige empirische Erkenntnisse generiert, sondern auch theoretischkonzeptionelle Zugänge aus der Betrachtung der Wohnungsfrage im weiteren Sinne entwickelt wurden.

Mit dem Einsetzen massiver Suburbanisierungsprozesse im westlichen Europa ab den 1950er-Jahren beginnt auch eine räumliche Aufweitung der Wohnungsfrage, die nun das (vormals ländliche) Stadtumland in den Blick nimmt. In diesem Zusammenhang diskutieren (seitdem) u. a. Geograph*innen die Gründe und Auswirkungen von Wanderungsbewegungen von Einwohner*innen ins Stadtumland. Der Urbanist Thomas Sieverts hat hierfür den Begriff der „Zwischenstadt“ geprägt, um auf die baulichen wie auch soziopolitischen Veränderungen hinzuweisen. Die Rolle des Eigenheims als standardisierte Wohnform Suburbias wird intensiv betrachtet. Das Wohnideal vom Haus im Grünen wird zum Synonym einer auf Konsum ausgerichteten kapitalistischen Reproduktionsweise, die vor allem im westlichen Europa und in Nordamerika zum dominanten automobilzentrierten Modell urbaner Entwicklung wird. „Auf der anderen Seite“ wird (in den Städten) in den 1950 er bis 1980 er-Jahren versucht, die Wohnungsfrage vor allem durch den Bau von Großwohnsiedlungen zu lösen. Dieser Lösungsansatz wird sowohl im östlichen als auch im westlichen Europa gewählt (Arch+2011). Die Intentionen der jeweiligen Wohnungspolitiken, die sich hinter dieser Form funktionalen Bauens verbergen, sind jedoch unterschiedlich. Während in Ländern des westlichen Europas Großwohnsiedlungen als sozialer Wohnbau deklariert werden und häufig (nur) für einkommensschwache Haushalte konzipiert sind, stehen Großwohnsiedlungen im östlichen Europa breiten gesellschaftlichen Schichten zur Verfügung. Die konkrete Umsetzung ruft aber in beiden Fällen 
sowohl in urbanistischer als auch sozialräumlicher Hinsicht Kritik hervor. Hinzukommt, dass sich die öffentliche Hand in Ost und West ab den 1990erJahren aus der Instandhaltung und Weiterentwicklung der Großwohnsiedlungen zurückzieht. Somit kommt es ab den 2000er-Jahren zu sozialräumlichen Verwerfungen. Großwohnsiedlungen erfahren vielfach Schrumpfungsprozesse. Dieses Schlagwort beschreibt den Zusammenhang von demographischem Wandel, Deindustrialisierung und Privatisierung, der die Wohnungsfrage in vielen europäischen Stadtregionen bestimmt (Haase et al. 2016).

Aktuell wird die räumliche Dimension der Wohnungsfrage in vielen urbanen Kontexten vor allem anhand der Themen "Gentrifizierung" und "Segregation" diskutiert. Gentrifizierung und Segregation sind sozialräumliche Prozesse, die an unterschiedlichen Orten weltweit untersucht werden, wodurch mittlerweile eine enorme Bandbreite an Wissen zu unterschiedlichen Formen und Ausprägungen entstanden ist (Lees \& Phillips 2018). Beide Prozesse stehen zudem für eine ungleiche Entwicklung, die, wie im Fall von Gentrifizierung, zu kleinräumigen Auf- und Abwertungsprozessen führt, die Verdrängung von Bewohner*innen nach sich zieht und Stadtquartiere auch abseits der Einwohner*innenstruktur maßgeblich verändert.

\section{4 Ökologische Dimension}

Wenngleich der sozialpolitische Fokus die (sozialwissenschaftliche) Betrachtung der Wohnungsfrage dominiert und teilweise sogar soziale und ökologische Belange gegeneinander ausgespielt werden, reicht ein Blick in das bereits mehrfach zitierte Beispiel des Industriezeitalters, um die Zusammenhänge von sozialer und ökologischer Dimension der Wohnungsfrage sichtbar zu machen (Vollmer \& Michel 2020: 163). Rauchende Schlote der Industrieanlagen, die ungefiltert Abgase ausstoßen, verunreinigte Abwässer, die Flüsse stark belasten sowie Enge, Lärm und Schmutz in Wohnvierteln aufgrund fehlender Stadt- und Wohnungspolitik, waren Kennzeichen vieler industrialisierter Städte zu Beginn des 20. Jahrhunderts. Folglich wurden die „besseren“ Wohnviertel häufig in den Westlagen europäischer Städte errichtet, womit die enge Verbindung von sozialer Segregation und ökologischen Rahmenbedingungen sichtbar wird.

Weitere Aspekte der (sozial)ökologischen Dimension der Wohnungsfrage zeigen sich aktuell vor allem bei der Errichtung von Wohnraum. Nachhaltiges Bauen mit ökologischen Materialien, reduzierte Flächenversiegelung, geringer Flächen- und Energieverbrauch, veränderbare, modulare Wohnformen sowie
Nachverdichtung sind zentrale Themen in dieser Hinsicht. Ein Beispiel, das die sozial-ökologische Dimension konkret zeigt, sind energetische Sanierungen von Wohnraum. ${ }^{14}$ Seit einigen Jahren ist die Energieeffizienz von Wohngebäuden ein zentraler Bestandteil europäischer und nationaler Förderpolitiken (Großmann 2020: 199). Kaum ein offizieller Bericht kommt ohne den Hinweis aus, dass energetische Sanierungen ressourcenschonend wirken und die Energiekosten für die Bewohner*innen sinken lassen. Vor allem der letztgenannte Aspekt wird in der sozialwissenschaftlichen Energiearmutsforschung mittlerweile kritisch betrachtet. Es gibt diverse Studien, die aufzeigen, dass vor allem einkommensschwache Haushalte grundsätzlich ressourcenschonend und teilweise sogar gesundheitsgefährdend sparsam leben, so dass preisintensive Sanierungen für sie primär eine enorme finanzielle Belastung und keine Entlastung darstellen (Brunner et al. 2017). Darüber hinaus werden energetische Sanierungen häufig pauschal durchgeführt, ohne dass der vorherige Energieverbrauch oder soziale Parameter einbezogen werden. In immobilienökonomischer Hinsicht wirken energetische Sanierungen als Preistreiber, da sie bspw. in der BRD mittels Modernisierungsumlage auf die Mieter*innen umgelegt werden (können), so dass sich innerhalb sehr kurzer Zeit die Sanierung für die Vermieter*innen amortisiert. Die finanzielle Belastung für die Mieter*innen ist erheblich. Die oftmals abrupten Mieterhöhungen mittels energetischer Sanierungen werden zudem bewusst als Verdrängungswerkzeug von Eigentümer*innen bzw. Investor*innen eingesetzt, wie Studien belegen. Sie sind somit ein „Katalysator für Segregation“ (Großmann 2020: 200). An dieser Stelle soll nicht der Eindruck entstehen, dass energetische Sanierungen ein überflüssiges Instrument sind. Ganz im Gegenteil! Sie sind auch angesichts der Klimakrise und des Ressourcenverbrauchs in Städten von großer Bedeutung. Allerdings gehen sie häufig zu Lasten einkommensschwacher Haushalte, weisen sozialpolitische Lücken in ihrer rechtlichen Konstruktion auf und verkennen grundsätzlich die Kräfteverhältnisse auf Wohnungsmärkten. Diese Schieflagen bedürfen gerade vor dem Hintergrund anstehender Modernisierungswellen, die insbesondere im öffentlichen Wohnbau der Nachkriegszeit in den nächsten Jahren anstehen, aufmerksamer und engagierter Forschung. Schlussendlich zeigt sich an diesem Beispiel, dass die Wohnungsfrage immer auch Gerechtigkeitsfragen inkludiert, die in ihrer Komplexität erörtert werden müssen, damit eine nachhaltige „Lösung“ der Wohnungsfrage möglich wird.

14 Einen fundierten Überblick zu diesem Thema gibt der Artikel von Großmann (2020). 


\section{Die Wohnungsfrage am Beispiel von Airbnb und Co.}

Wie lassen sich die aufgezeigten Dimensionen nun konkret anwenden? Welchen Erkenntnisgewinn ermöglichen sie bei der Erörterung spezifischer Wohnungsfragen? Hierzu gehe ich im Folgenden näher auf Kurzzeitvermietungen ein. Kurzzeitvermietungen (engl. short-term rentals) sind ein intensiv diskutiertes Thema der interdisziplinären Stadtforschung (CocolaGant 2016; Bianchi 2018; Armas-Díaz et al. 2021). Insbesondere Airbnb als dominanter Akteur, auf dessen Plattform Millionen von Kurzzeitvermietungsangeboten zu finden sind, wird zunehmend (auch in der Öffentlichkeit) kritisch betrachtet und u. a. für die Erhöhung von Wohnpreisen und die Verknappung von Wohnraum mitverantwortlich gemacht (Book \& Mingels 2020). Gleichzeitig ist Airbnb nicht nur ein global agierender Akteur, der die Wohnungsfrage in vielen Städten mit beeinflusst; Airbnb ist auch ein Beispiel eines neuen Plattformkapitalismus bzw. Plattformurbanismus, bei der gesellschaftliche Entwicklungen zusehends von und über Plattformen organisiert werden und somit neue Macht- und Kräfteverhältnisse entstehen (Langley \& Leyshon 2017).

\subsection{Zur politischen Ökonomie von Kurzzeitver- mietungen}

Die Narrative könnten nicht unterschiedlicher sein. Auf der einen Seite Airbnb, das sich gerne als Vorzeigebeispiel einer „kollaborativen“ Ökonomie präsentiert und stets auf seine Ursprünge als kleines Start-Up hinweist, aus dem mittlerweile eine weltumspannende "sharing economy of housing “ entstanden ist. ${ }^{15}$ Auf der anderen Seite gibt es eine stetig wachsende Zahl an wissenschaftlichen Studien, die Kurzzeitvermietung im Allgemeinen und Airbnb im Besonderen als eine neue Form der Kommodifizierung des Wohnens demaskieren (Wachsmuth \& Weisler 2018; CocolaGant \& Gago 2019; Grisdale 2019; Yrigoy 2019). In der Tat ist von dem kleinen Start-up, das übrigens bereits in seinen Anfängen Millionen an Wagniskapital (englisch venture capital) eingesammelt hat und somit schnell expansiv wachsen konnte, schon recht lange nicht mehr viel übrig (Srnicek 2017). Mitten in der Corona-Pandemie gelang Airbnb im November 2020 sogar der größte US-Börsengang des Jahres. Das Unternehmen war am Ende des ersten Handelstages über 100 Milliarden Dollar wert. ${ }^{16}$ Bereits diese Summen

\footnotetext{
15 siehe https://news.airbnb.com/about-us/ (13.04.2021)

16 siehe https://www.handelsblatt.com/finanzen/maerkte/aktien/online-zimmervermittler-fulminantes-debuet-airbnb-aktielegt-beim-boersengang-mehr-als-100-prozent-zu/26707102. html?ticket=ST-766959-dDsvhedAbb9pLlfKfy WZ-ap1
}

verdeutlichen, dass sich hinter der Vermittlung von Wohnraum ein profitables Geschäftsmodell verbirgt, bei dem der vielfach bemühte Sharing-Gedanke zumindest kritisch hinterfragt werden muss.

\section{Kurzzeitvermietung als Element einer Finanzialisie- rung des Wohnens}

Das dynamische Wachstum von Kurzzeitvermietungen ist eng verknüpft mit der Wirtschafts- und Finanzkrise von 2008. Im Zuge dieser globalen Krise ist Wohnen zu einem lukrativen Betätigungsfeld für anlagesuchendes Kapital geworden, da hiermit u.a. sichere Renditen verbunden sind. Investitionsflüsse unterschiedlicher Destination wurden in urbane Räume gelenkt (Alexandri \& Janoschka 2020). Hinzukommt, dass der globale Städtetourismus ab 2008 einen enormen Aufschwung genommen hat. Die Übernachtungszahlen sind in vielen urbanen Tourismusdestinationen sprunghaft gestiegen und haben zu einer Touristifizierung ${ }^{17}$ beigetragen, die lange Zeit von der öffentlichen Hand in vielen Städten forciert wurde (Gebhardt 2017). In diesem Kontext bilden Kurzzeitvermietungen eine Schnittstelle zwischen den Akkumulationsfeldern Wohnen und Städtetourismus; eine Schnittstelle, die es in dieser Form vorher nicht gab. Es hat sich in der Tat ein „neuer Wohnungsteilmarkt“ etabliert. Was aber macht diesen „neuen Wohnungsteilmarkt" ökonomisch attraktiv?

Aus einer empirischen Blickrichtung zeigen mittlerweile eine Vielzahl unterschiedlicher Studien und diverse Analyseportale, dass der „Airbnb-Markt“ vor allem durch das Angebot ganzer Wohnungen oder Häuser bestimmt wird. ${ }^{18}$ Es geht also nicht um die Bereitstellung der Wohnzimmercouch oder ungenutzter Wohnräume. Darüber hinaus bestimmen oftmals kommerzielle Akteure den Markt (Cocola-Gant \& Lopez-Gay 2020). ${ }^{19}$ Dies sind einerseits transnational agierende (institutionelle) Investor*innen, die Wohnraum zum Zwecke der Kurzzeitvermietung erwerben und diesen über spezialisierte Immobilienagenturen betreuen und managen (Katsinas 2021). Andererseits gibt es einen Mix aus privaten Eigentümer*innen - der bis weit in die Mittelklasse reicht - der die

(08.04.2021)

17 Touristifizierung beschreibt den Veränderungsdruck, mit dem Tourismus auf unterschiedliche urbane Quartierte wirkt und die damit einhergehende Ausrichtung der urbanen Infrastruktur auf den Städtetourismus.

18 http://www.insideairbnb.com (12.04.2021)

19 Zwar gibt es in vielen Städten eine signifikante Zahl von nicht-kommerziellen Anbieter*innen, die ihre eigene Unterkunft anbieten. Allerdings prägen diese Akteure häufig nicht das Marktgeschehen bzw. sind einem zunehmenden Professionalisierungsund Kommerzialisierungsdruck auch seitens der Plattformbetreiber wie Airbnb ausgesetzt (Smigiel et al. 2020). 
Ware Wohnen für sich entdeckt hat und diese nun profitabel anlegen will (Semi \& Tonetta 2020). Grisdale (2019) bezeichnet dies als das Aufkommen eines neuen, unternehmerischen Vermieter*innentyps. Alle genannten Akteure vereint neben der Aussicht auf (Mehr)Einnahmen, dass sie die Flexibilität von Kurzzeitvermietungen schätzen (Cocola-Gant 2020, Smigiel et al. 2020). Flexibilität bedeutet hierbei, dass die Anbieter*innen von Kurzzeitvermietungen ihr „Produkt" je nach Marktlage zeitlich und inhaltlich anpassen, um den jeweils höchstmöglichen Ertrag zu erzielen. Dies ist ein wesentlicher Unterschied zur klassischen Langzeitmiete und ein strukturelles Argument, warum short-term rentals klassische Mietformen verdrängen (siehe Kapitel 4.2).

Eine zweite konzeptionelle Perspektive schließt an den letztgenannten Gedanken an. Aus der Gentrifizierungsforschung ist der Erklärungsansatz der Ertragslücke (Rent-Gap) bekannt. Neil Smith hat diesen wegweisenden politökonomischen Erklärungsansatz in den 1970er-Jahren entwickelt, um zu zeigen, wie Investition und Desinvestition (Stadt)Räume prägen und sozialräumliche Prozesse bestimmen (Smith 1979). Kurzzeitvermietungen sind aus dieser Sicht für die Vermieter*innen strukturell attraktiv, da sie kurzfristige, enorme Gewinnsteigerungen aus dem Vermietungsgeschäft in Aussicht stellen. Konkret geht es um die Differenz (Ertragslücke) zwischen den aktuellen Einnahmen (bspw. vor Kurzzeitvermietung) - aktuelle, kapitalisierte Grundrente genannt - und den potenziell realisierbaren Einnahmen aus der Kurzzeitvermietung (potentielle Grundrente). Die sehr konkreten Gewinnaussichten der Kurzzeitvermietung setzen eine enorme Verwertungslogik in Gang, da hierfür kaum Investitionen notwendig sind (Yrigoy 2019). Mit vier Mausklicks lässt sich ein Angebot auf Airbnb \& Co. platzieren. Der Rent-Gap steigt folglich sehr dynamisch innerhalb kurzer Zeit an (Wachsmuth \& Weisler 2018: 1153). Neben der einfachen Handhabbarkeit hat die digitale Technologie zudem zu einer Sichtbarkeit vormals unbekannter Wohnungsmärkte geführt, die vor allem von professionellen Finanzmarktakteuren genutzt wird. Die Plattformisierung ${ }^{20}$ sorgt u.a. dafür, dass Wohnraum weltweit online besichtigt werden kann und von unterschiedlichen Akteuren zur Verfügung gestellt wird (Fields \& Rogers 2019). Außerdem sorgen einheitliche und vergleichbare Standards und Kategorien für eine Vergleichbarkeit der Angebote, was für einen stetigen Wettbewerbsdruck zwischen den Anbieter*innen sorgt.

\footnotetext{
$20 \quad$ Plattformisierung kann als Prozess verstanden werden, bei dem die Plattform zum dominanten ökonomischen Modell wird, über das Daseinsgrundfunktionen on- und offline organisiert und strukturiert werden (Helmond 2015: 1)
}

Zur politischen Ökonomie von Kurzzeitvermietungen gehört nicht zuletzt, dass mit Airbnb ein neuer mächtiger, globaler Player entstanden ist, der nicht nur mittels Technologie und Big Data Einfluss auf Stadt- und Wohnungspolitik nimmt. Zusätzlich setzt man bei Airbnb auf umfangreiche, multiskalare Lobbystrategien, die Entscheider*innen in Stadt- und Regionalpolitik ebenso adressieren wie nationale Ministerien oder europäische Ausschüsse und Gerichte (Aguilera et al. 2019; Smigiel 2020). Eine kürzlich veröffentlichte Studie zeigt außerdem, dass man auch auf neue Formen des Lobbyings setzt. Airbnb organisiert eigene Graswurzelbewegungen in Form von Home Sharing Clubs und Citizen Initiative, um öffentliche Debatten zu beeinflussen (Yates 2021). Das Ziel mag kurzfristig auf weniger Regulierung, geringe Steuern und Beschränkung ausgerichtet sein. Mittel- und langfristig verfolgt Airbnb das Ziel, sich als neue urbane Institution zu etablieren und den Rang eines regulierenden Akteurs zu erlangen, der sowohl öffentliche als auch zivilgesellschaftliche und privatwirtschaftliche Belange mitbestimmt (van Doorn 2019: 15).

\subsection{Geographien der Kurzzeitvermietung}

Die Auswirkungen der ökonomischen Rationalität von Kurzzeitvermietung betreffen nicht alle Städte und alle städtischen Räume gleichermaßen (Adamiak 2018). Eine hohe Dichte an Airbnb-Angeboten weisen jene Gebiete auf, in denen die Möglichkeit der Erzielung hoher Einnahmen besteht (Gutierrez \& Domenech 2020). Insbesondere innenstadtnahe Wohnviertel und Quartiere, die in räumlicher Nähe zu touristischen Hotspots liegen, weisen eine erhöhte „Airbnb-Dichte" auf (Gurran \& Phibbs 2017; Smigiel et al. 2020). Dies geht einher mit einem Phänomen das unter dem Schlagwort new urban tourism seit einigen Jahren in der Stadtforschung thematisiert wird (Füller \& Michel 2014). Dahinter verbirgt sich eine Ausweitung touristischer Aktivitäten abseits klassischer Sightseeing-Pfade in vormals weniger touristifizierte Stadtgebiete, womit eine strukturelle Veränderung der lokalen Ökonomie verbunden ist (Freytag $\&$ Glatter 2017). Kurzzeitvermietungen sind ein zentraler Bestandteil dieser neuen Form von Touristifizierung, die das Wohnangebot und die Wohnpreise in jenen Stadtvierteln verändern. Dementsprechend sind Studien wenig aussagekräftig, die ihren Fokus auf die gesamtstädtische Ebene richten und stadtweite Durchschnittsberechnungen anstrengen (BMWi 2018). Es ist gerade die räumliche Konzentration auf bestimmte Quartiere, die dort zu einer Erhöhung der Wohnpreise führt, wie bspw. eine aktuelle Studie am Beispiel Berlins zeigt (Duso et al. 2021). Airbnb und Co. sorgen in einigen Stadtteilen Berlins für eine Er- 
höhung der örtlichen Angebotsmieten um bis zu 46 Cent pro Quadratmeter (Duso et al. 2021).

Bedingen Kurzzeitvermietungen eine Verknappung des Wohnungsangebotes und kommt es folglich zu Verdrängungsprozessen? Mittlerweile gibt es einige Studien, die den ersten Teil der Frage anhand konkreter Fallbeispiele eindeutig mit Ja beantworten. Hierzu werden diejenigen Kurzzeitvermietungsangebote herangezogen, die als komplette Wohnungen dem Wohnungsmarkt über einen mehrmonatigen Zeitraum im Jahr nicht zur Verfügung stehen (Wachsmuth \& Weisler 2018; Grisdale 2019; Smigiel et al. 2020). Trotz sehr unterschiedlicher räumlicher Kontexte (New York, Toronto, Salzburg) wird deutlich, dass es große Übereinstimmungen gibt. Komplette Wohnungen bestimmen den Markt. D.h. 50-75\% der Kurzzeitvermietungen sind ganze Wohnungen. Ein Großteil dieser Wohnungen (bis zu 80\%) wird halboder sogar ganzjährig auf Airbnb und Co. angeboten und steht somit dem klassischen Wohnungsmarkt für Langzeitmieter*innen nicht zur Verfügung. Vermietet werden sie allerdings nicht ganzjährig. Die Ausnutzung variiert je nach Saison und Standort. Kurzzeitvermietungen führen demnach nicht nur zum Entzug von Wohnraum, sie sorgen auch für (temporären) Leerstand, was insbesondere in angespannten Wohnungsmärkten problematisch ist. Ungeachtet dessen übersteigen die Einnahmen aus Kurzzeitvermietungen den Ertrag aus Langzeitvermietungen um teilweise mehrere hundert Prozent, weshalb sich Vermieter*innen bewusst für den Wechsel von Lang- auf Kurzzeitmiete entscheiden (Wachsmuth \& Weisler 2018; Grisdale 2019; Smigiel et al. 2020).

Die hierdurch ausgelösten Verdrängungsprozesse sind vielfältig und gleichzeitig ein Thema, das weiterer Forschung bedarf. Studien aus Lissabon, einem Hotspot der Airbnb-Problematik, verdeutlichen die Heterogenität von Verdrängung (Cocola-Gant \& Gago 219). Verdrängung äußert sich einerseits im forcierten Wegzug aus der angestammten Wohnung und dem Wohnviertel. Andererseits ist dieser Form tourismus-induzierter Gentrifizierung ein zeitlich längerer Prozess vorgeschaltet, der auch eine sukzessive Verdrängung etablierter, lokaler Versorgungsinfrastruktur inkludiert. Insgesamt zeigt sich, dass Kurzzeitvermietungen sozialräumliche Ungleichheiten verstärken, die wiederum zeitverzögert auch in anderen Stadtvierteln ökonomische und soziale Verwerfungen auslösen.

\subsection{Wie lassen sich Kurzzeitvermietungen regulieren?}

Der Boom von Airbnb und Co. hat auch die Stadtpolitik zum Reagieren veranlasst; zumindest in den
Städten, die besonders viele Kurzzeitvermietungen aufweisen und hierfür ein Problembewusstsein entwickelt haben. ${ }^{21}$ Ein Auslöser sind häufig lokale Debatten, Proteste und Widerstand gegen extensive Touristifizierung (Colomb \& Novy 2017). Die entwickelten Antworten auf Kurzzeitvermietungen sind vielfältig. Zahlreiche Instrumente gab es bereits vor dem Aufkommen des Plattformurbanismus; andere wurden erst später entwickelt. Das Spektrum der planerischpolitischen Antworten reicht von zeitlichen Befristungen von Kurzzeitvermietungen, über verpflichtende Registrierungen / Lizenzen für Airbnb-Anbieter*innen bis hin zur Einrichtung von Stadtgebieten, in denen Kurzzeitvermietungen erlaubt/verboten werden (Nieuwland \& Van Melik 2018). Die Heterogenität planerisch-politischer Antworten resultiert nicht zuletzt aus teilweise sehr divergierenden Sichtweisen auf das Phänomen Kurzzeitvermietungen in den jeweiligen Stadtverwaltungen. Wie das Phänomen angegangen wird, hängt zudem davon ab, welche politischen Akteure das Thema diskursiv besetzen und voranbringen, wie Aguilera et al. in einer komparativen Studie am Beispiel von Barcelona, Mailand und Paris erläutern (2019).

Vor drei generellen Problemen stehen aber alle Kommunen: Erstens müssen die gewählten Restriktionen kontrolliert und sanktioniert werden. In einer Vielzahl von Städten fehlt es aber an Personal, um den erlassenen Beschränkungen nachzugehen. Zweitens können städtische Regulierungsversuche nur ein erster Ansatz sein. Gerade im Hinblick auf raumplanerische und steuerliche Aspekte ist es essentiell, regionale und nationale Gremien und Entscheidungsträger*innen mit einzubeziehen, wie Holzmannhofer für den österreichischen Kontext deutlich macht (Holzmannhofer 2021). Die Multiskalarität dieses Themas zeigt sich nicht zuletzt daran, dass Airbnb gerade auf der europäischen Ebene bisher erfolgreich stärkere Regulierungen verhindert hat. Für die EU Kommission ist Airbnb weiterhin ein Wegbereiter einer „kollaborativen Ökonomie“; ein Argument, das die politische Ökonomie dieses Phänomens ebenso vernachlässigt wie es ein rudimentäres Verständnis von Plattformkapitalismus offenbart (Smigiel 2020). Hiermit wären wir beim dritten Problem. Plattformökonomien basieren auf der Sammlung, Kombination und Verwertung von Big Data. Deren Sammlung ist kein Selbstzweck, sondern es geht um das zeitnahe Nutzen von Daten und um die Entwicklung neuer Geschäftsfelder (Fields \& Rogers 2019: 2). Daten von Vermieter*innen und Vermietungspraktiken sind es-

21 siehe https://www.amsterdam.nl/nieuwsarchief/persberichten/2019/persberichten-laurens-ivens/press-release-cities-alarmed-about/ (13.04.2021) 
sentieller Bestandteil des Prozesses. Dementsprechend defensiv gehen Airbnb und Co. mit den generierten Daten um. Die Herausgabe von Vermietungsdaten in größerem Umfang ist nur in sehr wenigen Fällen nach lokalen gerichtlichen Auseinandersetzungen erfolgt. Auf europäischer Ebene hat der Europäische Gerichtshof basierend auf der Dienstleistungsrichtlinie 2000/31 bisher für Airbnb entschieden (Gerichtshof der EU 2019). Eine Überarbeitung dieser Richtlinie findet gerade statt. Es ist zu hoffen, dass der neue Digital Service Act den Einfluss von Plattformökonomien klarer erkennt und gewichtet, wodurch auch die Arbeit der öffentlichen Hand erleichtert würde.

\section{4 ...und wie steht es mit der Nachhaltigkeit?}

Eine zentrale Herausforderung der aktuellen Wohnungsforschung ist es nachhaltige Lösungen oder zumindest Ansätze zu entwickeln. Nachhaltigkeit als Konzept und Leitparadigma beruht auf drei ,thematischen "Säulen. Vor diesem Hintergrund ließe sich fragen, inwiefern Kurzzeitvermietungen ökologisch, sozial und ökonomisch nachhaltig sind? Diese Frage lässt sich nicht abschließend beantworten und würde auch den Rahmen dieses Beitrags übersteigen. Daher beschränke ich mich zum Abschluss auf einige Gedanken.

Hinsichtlich der sozialen Dimension haben die bisherigen Ausführungen verdeutlicht, dass die Anbieter*innen von Airbnb und Co, nur einen kleinen Ausschnitt (urbaner) Bevölkerungen repräsentieren. Es finden sich abseits von Immobilienunternehmen zwar jede Menge "gewöhnlicher Leute" unter den Airbnb-Anbieter*innen. Dominant sind jedoch Akteure aus der gehobenen Mittelschicht, die zudem vielfach über ausreichend ökonomisches Kapital verfügen (Roelofsen 2018). Hinzukommen zahlreiche sichtbare und unsichtbare „Eintrittsbarrieren“, die ungeachtet der einfachen technischen Einrichtung eines Airbnb-Profils, über den Erfolg mitbestimmen. Kulturelles und soziales Kapital im Bourdieuschen Sinne ist notwendig, um ein attraktives Angebot zu schaffen, das auch Nachfrage generiert. Eigentum oder eine gewisse Kontrolle über die Ware Wohnen ist ebenfalls eine Voraussetzung, die von zentraler Bedeutung ist und exkludierend wirkt (Crommelin et al. 2018). Aus einer nachhaltigen Perspektive ist es außerdem relevant, dass Airbnb und Co. vielerorts die Wohnpreise steigen lassen. Das Wohnraumangebot wird insgesamt verringert, was besonders für einkommensschwächere Einwohner*innen problematisch ist. Kurzzeitvermietungen sorgen also eher für eine $\mathrm{Zu}$ nahme sozialräumlicher Ungleichheit als dass sie soziale Gerechtigkeit fördern.

Der Gedanke des Teiles bzw. eine bessere und effizientere Nutzung von Wohnraum ist ein Mythos, der weiterhin Airbnb und Co. umgibt (Smigiel 2020). Bereits ein Blick auf das Geschäftsmodell „Kurzzeitvermietung“ lässt Zweifel aufkommen, ob hiermit eine ökologisch nachhaltige Nutzung von Wohnraum verbunden ist. Es findet vielmehr eine Kommodifizierung und Globalisierung des Wohnens statt. Kurzzeitvermietungen sind außerdem gekoppelt an intensive Reisetätigkeiten und tragen somit zu steigender Mobilität bei. Und letztlich basiert diese „Sharing Economy" auf dem Gedanken des Wettbewerbs und der Generierung ökonomischen Mehrwerts aus dem "Teilen des Wohnraums“, was nicht auf ein Modell im Sinne sozialer, ökonomischer und ökologischer Nachhaltigkeit schließen lässt.

\section{$5 \quad$ Ein kurzer Ausblick}

Die Corona-Pandemie hat vieles - zumindest kurzfristig - verändert. Eine Konstante ist jedoch geblieben: Die Wohnungsfrage bleibt weiterhin aktuell. Dies lässt sich an stetig steigenden Boden- und Wohnpreisen ebenso ablesen, wie an der Bedeutung des Wohnorts für gesellschaftliche Teilhabe und Wohlergehen. Zwar gibt es zum Konnex Risiko einer Corona-Erkrankung und dem Wohnort/Wohnumfeld bisher nur vereinzelte Untersuchungen. Allerdings kommen diese ungeachtet unterschiedlicher Untersuchungskontexte (Deutschland, Indien, USA etc.) größtenteils zu einem ähnlichen Fazit (Klugman \& Moore 2020: 7 ff.; Plümper \& Neumayer 2020). Konkret bedeutet dies: Je länger die Pandemie dauert, desto verstärkender wirken sich sozialräumliche Ungleichheiten auf das Risiko einer Erkrankung, schwerer Krankheitsverläufe etc. aus (Gräff 2021). In der Folge vertiefen sich wiederum die sozialräumlichen Ungleichheiten.

Im vorliegenden Beitrag habe ich die Wohnungsfrage am Beispiel von Airbnb und Co. konkretisiert. Ebenso wie die Wohnungsfrage aktuell bleiben wird, sind auch Kurzzeitvermietungen und der digitale Plattformurbanismus im Allgemeinen zukunftsprägende Themen. Die Hintergründe und Argumente für diese These habe ich in Kapitel 4 dargelegt. Drei Aspekte scheinen mir abschließend nochmals wichtig zu betonen. Die Plattformisierung hat strukturelle Veränderungen ausgelöst, die sich durch die gegenwärtige Krise zumindest teilweise vertieft haben. Im Hinblick auf Airbnb und Co. lässt sich diesbezüglich festhalten, dass hier ein neues Modell der Kommodifizierung des Wohnens geschaffen wurde, das Flexibilität und Wachstum in einer besonderen Weise vereint. Flexibilität ist ein entscheidendes Motiv, das die ökonomische Attraktivität von Kurzzeitvermietungen ausmacht. Kurzzeitvermietungen stehen in diesem Zusammenhang für eine flexible, fortwähren- 
de Umwandlung einer Grunddaseinsfunktion in einen finanziellen Vermögenswert mit all ihren (negativen) Konsequenzen (Armas-Díaz et al. 2021: 13). Der Aspekt des Wachstums ist hierbei von ebenso großer Bedeutung, da die Mehrzahl der Plattformen grundsätzlich darauf ausgerichtet ist, schnell und extensiv zu wachsen, wie diverse Studien belegen (Langley \& Leyshon 2017).

Abseits fachwissenschaftlicher Fragen zu denen es langfristiger (auch geographischer) Forschungen bedarf, hat der Beitrag deutlich gemacht, dass die Wohnungsfrage ein gesellschaftliches Dauerthema ist, zu dem eine multidimensionale Perspektive notwendig erscheint, um ein umfassendes Verständnis einerseits und nachhaltige Lösungen andererseits zu generieren (Schönig \& Vollmer 2020: 7). Die Wohnungsfrage ist aber auch ein Querschnittsthema und somit gerade für den schulischen Bereich besonders relevant, da es viele alltagsweltliche und gesellschaftliche Thematiken verbindet. Hierfür kann der Beitrag an dieser Stelle keine vertiefenden Anregungen geben. Dies wäre aber sicherlich ein spannendes und wichtiges Anliegen, zu dem es nicht zuletzt analytischer und methodischer Überlegungen aus der Perspektive der Fachdidaktik bedarf.

\section{Literatur}

Aalbers, M. B. (2019): Introduction to the Forum; From Third to Fifth-Wave Gentrification.In: Tijdschrift voor Economische en Sociale Geografie 110(1). S. 1-11.

Adamiak, C. (2018): Mapping Airbnb supply in European cities. In: Annals of Tourism Research 71. S. 67-71.

Aguilera, T., F. Artioli, \& C. Colomb (2019): Explaining the diversity of policy responses to platform-mediated shortterm rentals in European cities: A comparison of Barcelona, Paris and Milan. In: Environment and Planning A. DOI: https://doi.org/10.1177/0308518x19862286

Aigner, A. (2020): What's wrong with investment apartments? On the construction of a 'financialized' rental investment product in Vienna. In: Housing Studies. DOI: https://10.1080/02673037.2020.1806992

Alexandri, G. \& M. Janoschka (2020): 'Post-pandemic' Transnational Gentrifications: A Critical Outlook. In: Urban Studies 57(15). S. 3202-3214.

Amann, W. \& K. Lugger (2016): Österreichisches Wohnhandbuch. StudienVerlag, Innsbruck.

Arch+ (2011): Planung und Realität. Strategien im Umgang mit den Großwohnsiedlungen. In: Arch+ 203.

Aring, J. \& G. Herfert (2001): Neue Muster der Wohnsuburbanisierung. In: Brake, K., J. S. Dangschat \& G. Herfert (Hrsg.): Suburbanisierung in Deutschland. Aktuelle Tendenzen. Springer, Wiesbaden. S. 43-56.

Armas-Díaz, A., C. Smigiel \& M. Janoschka (2021): Stadt|Tourismus kritisch hinterfragt - Zur Einführung in ein aufstrebendes humangeographisches Forschungsfeld. In: Berichte. Geographie und Landeskunde 94(1). S. 3-19.

Bianchi, R. (2018): The Political Economy of Tourism Development: A Critical Review. In: Annals of Tourism Research 70. S. 88-102.

Book, S. \& G. Mingels (2020): Der Weltvermieter. In: Der Spiegel, 50/2020. S. 72-74.

Brunner, K. H., A. Christanell \& S. Mandl (2017): Energiearmut in Österreich: Erfahrungen, Umgangsweisen und Folgen. In: Großmann, K., A. Schaffrin \& C. Smigiel (Hrsg.): Energie und soziale Ungleichheit. Zur gesellschaftlichen Dimension der Energiewende in Deutschland und Europa. Springer, Wiesbaden. S. 131-156.

Bundesministerium für Wirtschaft und Energie (2018): Sharing Economy im Wirtschaftsraum Deutschland. Berlin.

Bürkner, H. J. (2018): Segregation. In: Belina, B., M. Naumann \& A. Strüver (Hrsg.): Handbuch Kritische Stadtgeographie. Westfälisches Dampfboot, Münster. S. 158-163.

Chorherr, C. (2019): „Man ist nur dann ohnmächtig, wenn man glaubt es zu sein “. In: dérive - Zeitschrift für Stadtforschung 74. S. 11-17.

Claßen, G. \& C. Zander (2010): Handel mit Mietwohnungsportfolios in Deutschland Umfang und Auswirkungen vor dem Hintergrund von Internationalisierung und Professionalisierung. In: Informationen zur Raumentwicklung 5-6. S. 377-390.

Cocola-Gant, A. (2016): Holiday rentals: the new gentrification battlefront. In: Sociological Research Online21(10). DOI: https://doi.org/10.5153/sro.4071

Cocola-Gant, A (2020): Short-term rentals, Covid-19 and platform capitalism. http://www.albasud.org/blog/ en/1220/short-term-rentals-covid-19-and-platform-capitalism (13.04.2021)

Cocola-Gant, A. \& A. Gago (2019): Airbnb, buy-to-let investment and tourism-driven displacement: A case study in Lisbon. In: Environment and Planning A. DOI: https://doi.org/10.1177/0308518X19869012

Cocola-Gant, A. \& A. Lopez-Gay (2020): Transnational Gentrification, Tourism and the Formation of 'Foreign Only’ Enclaves in Barcelona. In: Urban Studies 57(15). S. 3025-3043.

Colomb, C. \& J. Novy (2017): Protest and resistance in the tourist city. Contemporary geographies of leisure, tourism and mobility. Routledge, London.

Crommelin, L., L. Troy, C. Martin \& C. Pettit (2018): Is Airbnb a Sharing Economy Superstar? Evidence from Five Global Cities. In: Urban Policy Research 36. S. 429-444.

Deutscher Bundestag (2019): Recht auf Wohnen. Ausgestaltung und Rechtswirkung in den Verfassungen der Bundesländer und der EU-Mitgliedstaaten.

Duso, T., C. Michelsen, M. Schäfer \& K. Tran (2021): Durch Airbnb-Vermietungen steigen in Berlin die Mieten. In: DIW Wochenbericht, 7/2021. S. 95-102. 
Egner, B. \& D. Rink (2020): Lokale Wohnungspolitik. Beispiele aus deutschen Städten. Bd. 4. Nomos, Baden-Baden.

Elsinga, M., M. Stephens \& T. Knorr-Siedow (2014): The privatisation of social housing. Three different pathways. In: Scanlon, K., C. Whitehead \& M. F. Arrigoitia (Hrsg.): Social Housing in Europe. Wiley Blackwell, Oxford. S. 389-414.

Engels, F. (1970 [1845]): Die Lage der arbeitenden Klasse in England. Marx-Engels-Werke 2. Berlin. S. 225-506.

Fields, D. \& D. Rogers (2019): Towards a Critical Housing Studies Research Agenda on Platform Real Estate, Housing, Theory and Society. DOI: https://doi.org/10. 1080/14036096.2019.1670724

Fezer, J., N. Hirsch, W. Kuehn \& H. Peleg (2017): Wohnungsfragen. In: Fezer, J., N. Hirsch, W. Kuehn \& H. Peleg (Hrsg.): Wohnungsfrage. Matthes \& Seitz, Berlin. S. $10-17$.

Freytag, T. \& J. Glatter (2017): Touristifizierung städtischer Quartiere - Synergien und Konflikte zwischen tourismusgerechter Stadt und stadtgerechtem Tourismus: In: Geographische Zeitschrift 105(3-4). S. 163-166.

Füller, H. \& B. Michel (2014): 'Stop Being a Tourist!' New Dynamics of Urban Tourism in Berlin-Kreuzberg. In: International Journal of Urban and Regional Research 38(4). S. 1304-1318.

Gebhardt, D. (2017): Barcelona: Die Drosslung des Wachstumsmotors Tourismus? In: Geographische Zeitschrift 105(3-4). S. 225-248.

Gerichtshof der Europäischen Union (2019): France cannot require Airbnb to hold an estate agent's professional licence as it did not notify the Commission of that requirement in accordance with the Directive on electronic commerce In: Judgment in Case C-390/18 Airbnb Ireland, EU.

Gräff, F. (2021): Die geteilte Stadt. In: Taz - Die Tageszeitung, 30.5.2021. https://taz.de/Corona-und-soziale-Ung leichheit/!5771211\&s=veddel/

Grisdale, S. (2019): Displacement by disruption: shortterm rentals and the political economy of "belonging anywhere" in Toronto. In: Urban Geography. DOI: https://doi.org/10.1080/02723638.2019.1642714

Großmann, K. (2020): Gebäude-Energieeffizienz als Katalysator residentieller Segregation. In: sublurban - Zeitschrift für kritische Stadtforschung 8(1-2). S. 199-210.

Gurran, N. \& P. Phibbs (2017): When Tourists Move In: How Should Urban Planners Respond to Airbnb? In: Journal of the American Planning Association 83. S. 80-92.

Gutiérrez, A. \& A. Domènech (2020): Understanding the spatiality of short-term rentals in Spain: Airbnb and the intensification of the commodification of housing. In: Geografisk Tidsskrift - Danish Journal of Geography 120(2). S. 98-113.

Haase, A., M. Bernt, K. Großmann, V. Mykhenenko \& D. Rink (2016): Varieties of shrinkage in European cities. In: European Urban and Regional Studies 23(1). S. $86-102$.
Heeg, S. (2013): Wohnungen als Finanzanlage. Auswirkungen von Responsibilisierung und Finanzialisierung im Bereich des Wohnens. In: sublurban. Zeitschrift für kritische Stadtforschung 1(1). S. 75-99.

Helbig, M. \& S. Jähnen (2018): Wie brüchig ist die soziale Architektur unserer Städte? Trends und Analysen der Segregation in 74 deutschen Städten. In: WZB Discussion Paper P 2018-001. Berlin.

Helmond, A. (2015): The Platformization of the Web: Making Web Data Platform Ready. In: Social Media + Society. DOI: https://doi.org/10.1177/2056305115603080

Holm, A. (2011): Wohnung als Ware: zur Ökonomie und Politik der Wohnungsversorgung. In: Widersprüche Zeitschrift für sozialistische Politik im Bildungs-, Gesundheits- und Sozialbereich 31(121). S. 9-20.

Holm, A. (2020): Berlin: Mehr Licht als Schatten. Wohnungspolitik unter Rot-Rot-Grün. In: Egner, B. \& D. Rink (Hrsg.): Lokale Wohnungspolitik. Beispiele aus deutschen Städten. Bd. 4. Nomos, Baden-Baden. S. 43-64.

Holzmannhofer, F. (2021): Recht. Airbnb-Update. In: ÖGZ. Das Magazin des Österreichischen Städtebundes 2. S. 74-76.

Hubeli, E. (2020): Die neue Krise der Städte. Zur Wohnungsfrage im 21. Jahrhundert. Rotpunktverlag, Zürich.

Katsinas, P. (2021): Professionalisation of short-term rentals and emergent tourism gentrification in post-crisis Thessaloniki. Environment and Planning A. DOI: https://doi.org/10.1177/0308518X21988940

Kockelkorn, A. (2017): Wohnungsfrage Deutschland: Zurück in die Gegenwart. In: Fezer, J., N. Hirsch, W. Kuehn \& H. Peleg (Hrsg.): Wohnungsfrage. Matthes \& Seitz, Berlin. S. 106-143.

Klugman, J. \& Moore, M. (2020): COVID-19 has a postcode: How urban housing and spatial inequality are shaping the COVID-19 crisis. Research paper. New York City University, Center on International Cooperation (CIC).

Langley, P. \& A. Leyshon 2017: Platform Capitalism: The Intermediation and Capitalisation of Digital Economic Circulation. In: Finance and Society 3(1). S. 11-31.

Lees. L. \& M. Phillips (2018): Handbook of Gentrification Studies. Routledge, London.

Mayer, K. (2020): ... oder warum Boden kein Joghurt ist. In: Mayer, K., K. Ritter, A. Fitz \& AzW Wien (Hrsg.): Boden für alle. Park Books, Zürich. S. 62-83.

Moore, J. \& S. Schindler (2017): Eine Definition der Ungleichheit. In: Fezer, J., N. Hirsch, W. Kuehn \& H. Peleg (Hrsg.): Wohnungsfrage. Matthes \& Seitz, Berlin. S. 62-81.

Nieuwland, S. \& R. Van Melik (2018): Regulating Airbnb: how cities deal with perceived negative externalities of short-term rentals. In: Current Issues in Tourism 23. S. 811-825.

Padberg, S. \& M. Schraven (2015): „Warum Spanien ein paar Jahre reich war?" - Darf mit Wohnungen spekuliert werden? In: GW-Unterricht 139(3). S. 42-53. 
Parade, R. \& F. Heinzel (2020): Sozialräumliche Segregation und Bildungsungleichheiten in der Grundschule - eine Bestandsaufnahme. In: Zeitschrift für Grundschulforschung. DOI: https://doi.org/10.1007/s42278020-00080-w

Pistor, K. (2020): Der Code des Kapitals. Suhrkamp, Frankfurt am Main.

Plümper, T. \& E. Neumayer (2020): The pandemic predominantly hits poor neighbourhoods? SARS-CoV-2 infections and COVID-19 fatalities in German districts. In: The European Journal of Public Health 30(6). S. $1176-1180$.

Rink, D. \& L. Vollmer (2019): „Mietenwahnsinn stoppen!“ Netzwerke und Mobilisierungen der Mieter*innenbewegung in deutschen Großstädten. In: Forschungsjournal SozialeBewegungen 32(3).S.337-349.

Roelofsen, M. (2018): Exploring the Socio-Spatial Inequalities of Airbnb in Sofia, Bulgaria In: Erdkunde 72. S. 313-327.

Scanlon, K., C. Whitehead \& M. F. Arrigoitia (2014): Social Housing in Europe. Wiley Blackwell, Oxford.

Schönig, B. \& L. Vollmer (2020): Wohnungsfragen ohne Ende?! Interdisziplinäre Wohnungsforschung. Bd. 1. Transcript, Bielefeld.

Schönig, B. \& L. Vollmer (2020): Wohnungsfragen ohne Ende?! Sechs Thesen für eine interdisziplinäre Wohnungsforschung. In: Schönig, B. \& L. Vollmer (Hrsg.).: Wohnungsfragen ohne Ende?! Interdisziplinäre Wohnungsforschung. Bd. 1. Transcript, Bielefeld. S. 7-33.

Semi, G. \& M. Tonetta (2020): Marginal hosts. Short-term rentals suppliers in Turin, Italy. In: Environment and Planning A. DOI: https://doi. org/10.1177/0308518X20912435

Silomon-Pflug, F. (2018): Die Verwaltung der unternehmerischen Stadt (K)ein Thema in der geographischen Stadtforschung?! In: sublurban - Zeitschrift für kritische Stadtforschung 6(2-3). S. 49-68.

Smigiel, C. (2016): Raumproduktionen im südöstlichen Europa. Bd 24. Westfälisches Dampfboot, Münster.

Smigiel, C. (2020): Reflections over Regulating Airbnb and the Complexity and Agency of Platform Capitalism. In: Geographica Helvetica 75(3). S. 253-257.
Smigiel, C., A. Hof, K. Kautzschmann \& R. Seidl (2020): Ein Mixed-Methods-Ansatz zur Analyse von Kurzzeitvermietungen und ihren sozialräumlichen Auswirkungen am Beispiel der Stadt Salzburg. In: Raumforschung \& Raumordnung 78(2). S 153-170.

Smith, N. (1979): Toward a Theory of Gentrification A Back to the City Movement by Capital, not People. In: Journal of the American Planning Association 45(4). S. $538-548$.

Srnicek, N. (2017): Platform capitalism. Polity Press, Cambridge. Streimelweger,A.(2010): Wohnbauförderung:EineBestandsaufnahme. In: Wirtschaft Gesellschaft 36. S. 543-561.

van Doorn, N. (2019): A new institution on the block: On platform urbanism and Airbnb citizenship. In: New Media \& Society. DOI: https://doi. org/10.1177/1461444819884377

Van-Hametner, A., C. Smigiel, K. Kautzschmann \& C. Zeller (2019): Die Wohnungsfrage abseits der Metropolen: Wohnen in Salzburg zwischen touristischer Nachfrage und Finanzanlagen. In: Geographica Helvetica 74(2). S. 235-248.

Vollmer, L. \& B. Michel (2020): Wohnen in der Klimakrise. Die Wohnungsfrage als ökologische Frage: Aufruf zur Debatte. In: sublurban - Zeitschrift für kritische Stadtforschung 8(1-2). S. 163-166.

Wachsmuth, D. \& A. Weisler (2018): Airbnb and the rent gap: Gentrification through the sharing economy. In: Environment and Planning A 50. S. 1147-1170.

Whitehead, C. (2014): Social Housing in England. In: Scanlon, K., C. Whitehead \& M. F. Arrigoitia (Hrsg.): Social Housing in Europe. Wiley Blackwell, Oxford. S. 105-122.

Yates, L. (2021): The 'airbnb' movement for deregulation. https://research.ethicalconsumer.org/research-hub/ethical-consumption-review (10.4.2021)

Yrigoy, I. (2019): Rent gap reloaded: Airbnb and the shift from residential to touristic rental housing in the Palma Old Quarter in Mallorca, Spain. In: Urban Studies 56. S. 2709-2726.

Zeller, C., A. Van-Hametner, C. Smigiel \& K. Kautzschmann (2018): Wohnen in Österreich: von der sozialen Infrastruktur zur Finanzanlage. In: PROKLA. Zeitschrift für kritische Sozialwissenschaft 48(193), S. 597-616. 